\title{
Determinantes sociais de saúde e autoeficácia de mães/ cuidadores para prevenção da diarreia
} Social determinants of health and self-efficacy of mothers/caregivers for preventing diarrhea Determinantes sociales de salud y autoeficacia de madres/cuidadores para prevención de diarrea

Elizamar Regina da Rocha Mendes ${ }^{1}$ (D) https://orcid.org/0000-0002-1552-5622 Paulo Henrique Alexandre de Paula ${ }^{1}$ ib https://orcid.org/0000-0003-4583-2989

Kamila Ferreira Lima ${ }^{1}$ iD https://orcid.org/0000-0002-4554-3178

Paulo César de Almeida² (i) https://orcid.org/0000-0002-2867-802X

Patricia Neyva da Costa Pinheiro ${ }^{1}$ id https://orcid.org/0000-0001-7022-8391

Emanuella Silva Joventino Melo ${ }^{3}$ ic https://orcid.org/0000-0001-9786-5059

Lorena Pinheiro Barbosa ${ }^{1}$ io https://orcid.org/0000-0002-8006-7517

Como citar:

Mendes ER, Paula PH, Lima KF, Almeida PC, Pinheiro PN, Melo ES, et al. Determinantes sociais de saúde e autoeficácia de mães/ cuidadores para prevenção da diarreia. Acta Paul Enferm. 2021;34:eAPE000615.

DOI http://dx.doi.org/10.37689/actaape/2021A0000615

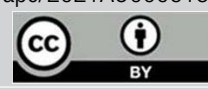

Descritores

Diarreia infantil; Determinantes sociais da saúde; Autoeficácia; Promoção da saúde; Prevenção de doenças

Keywords Diarrhea, infantile; Social determinants of health; Self efficacy; Health promotion; Disease prevention

Descriptores

Diarrea infantil; Autoeficacia; Determinantes sociales de la salud; Promoción de la salud; Prevención de enfermedades

$$
\begin{array}{r}
\text { Submetido } \\
27 \text { de Março de } 2020 \\
\text { Aceito } \\
1 \text { de Março de } 2021
\end{array}
$$

Autor correspondente

Elizamar Regina da Rocha Mendes E-mail: elizamarregina@hotmail.com

\section{Resumo}

Objetivo: Verificar associação entre os determinantes sociais de saúde e os níveis de autoeficácia de mães ou cuidadores para prevenção da diarreia infantil.

Métodos: Estudo transversal realizado com 363 mães ou cuidadores de crianças menores de cinco anos, acompanhadas em unidade de Atenção Primária à Saúde. Para coleta de dados, utilizaram-se o formulário de caracterização sociodemográfica e a Escala de Autoeficácia Materna para Prevenção da Diarreia Infantil. Realizou-se o teste Qui-Quadrado e como medida de associação a razão de chances.

Resultados: Verificou-se que mães ou cuidadores com idades de 15 a 29 anos tiveram 1,6 vezes mais chances de apresentarem autoeficácia moderada, com significância estatística para o determinante individual "faixa etária" ( $p=0,017)$. Os participantes com determinantes intermediários "destino do lixo" e "origem da água do domicílio" inadequados tiveram, respectivamente, 3,5 e 2,8 vezes mais chances de apresentarem autoeficácia moderada.

Conclusão: 0 Determinante Social de Saúde "faixa etária" aumenta a chance de mães ou cuidadores apresentarem autoeficácia moderada para prevenção da diarreia infantil. Portanto, cabe aos enfermeiros uma maior ênfase em orientar as mães ou cuidadores, principalmente os mais jovens sobre a prevenção da diarreia e realizar ações educativas que visem aumentar a autoeficácia destes.

\section{Abstract}

Objective: To verify the association between the social determinants of health and the levels of self-efficacy of mothers or caregivers to prevent childhood diarrhea.

Methods: This is a cross-sectional study carried out with 363 mothers or caregivers of children under five years old, followed up in a Primary Health Care unit. For data collection, the sociodemographic characterization form and the Maternal Self-Efficacy Questionnaire for Prevention of Infantile diarrhea. The Chi-Square test was performed, and as a measure of association, odds ratio.

Results: It was found that mothers or caregivers aged 15 to 29 years were 1.6 times more likely to have moderate self-efficacy, with statistical significance for the individual determinant "age group" $(p=0.017)$. Participants with inadequate intermediate determinants "waste destination" and "home water source" were 3.5 and 2.8 times more likely to have moderate self-efficacy, respectively.

Conclusion: The social determinant of health "age group" increases the chance of mothers or caregivers showing moderate self-efficacy to prevent childhood diarrhea. Therefore, nurses should place a greater emphasis on guiding mothers or caregivers, especially younger ones, on the prevention of diarrhea and carrying out educational actions aimed at increasing their self-efficacy. 


\section{Resumen}

Objetivo: Verificar la relación entre los determinantes sociales de salud y los niveles de autoeficacia de madres o cuidadores para la prevención de diarrea infantil.

Métodos: Estudio transversal realizado con 363 madres o cuidadores de niños menores de cinco años, atendidas en una unidad de Atención Primaria de Salud. Para la recopilación de datos se utilizó el formulario de caracterización sociodemográfica y la Escala de Autoeficacia Materna para la Prevención de la Diarrea Infantil. Se realizó la prueba $\chi^{2}$ de Pearson y, como medida de asociación, la razón de momios.

Resultados: Se verificó que las madres o cuidadores con edad de 15 a 29 años tuvieron 1,6 veces más chances de presentar autoeficacia moderada, con significación estadística del determinante individual "grupo de edad" ( $p=0,017)$. Los participantes con determinantes intermedios "destino de la basura" y "origen del agua del domicilio" inadecuados tuvieron más probabilidades de presentar autoeficacia moderada, 3,5 y 2,8 respectivamente.

Conclusión: El determinante social de salud "grupo de edad" aumenta la probabilidad de que madres o cuidadores presenten autoeficacia moderada para la prevención de la diarrea infantil. Por lo tanto, corresponde a los enfermeros hacer hincapié en orientar a las madres o cuidadores, principalmente los más jóvenes, sobre la prevención de la diarrea y realizar acciones educativas con el objetivo de aumentar su autoeficacia.

\section{Introdução}

A diarreia continua sendo a segunda principal causa de morte na população infantil, embora seja possível de prevenir e tratar. Globalmente cerca de 525.000 óbitos de crianças menores de cinco anos são registrados a cada ano, com alta prevalência sobretudo em países menos desenvolvidos. ${ }^{(1)}$

No Brasil, durante o ano de 2019, foram registrados 46.816 casos de diarreia em crianças menores de cinco anos. ${ }^{(2)}$ Entre os meses de janeiro a julho de 2020, ocorreram 16.857 internaçôes por diarreia na mesma faixa etária. ${ }^{(3)}$ Em 2018, no Nordeste, foram registrados 151 óbitos de crianças menores de 4 anos, sendo que 21 destas crianças eram do Ceará. ${ }^{(3,4)}$ No mesmo estado, em 2019, ocorreram 2.329 casos de diarreia em menores de 4 anos. ${ }^{(4,5)}$

A diarreia pode estar relacionada a múltiplos fatores de ordem ambiental, socioeconômica e cultural, ou seja, a Determinantes Sociais da Saúde (DSS), ${ }^{(6)}$ que abrange as condiçôes de vida e de trabalho dos indivíduos e de grupos da população, bem como comportamentos em relaçáo aos cuidados à saúde. (7,8) $^{-1}$

No Brasil, a Comissão Nacional sobre Determinantes Sociais da Saúde adotou o modelo de Dalgren e Whitehead para orientar a organização de suas atividades, por ser considerado simples e de fácil visualização, organizado em círculos concêntricos, tendo como centro os indivíduos, envoltos por cinco camadas. A primeira camada é representada pelos determinantes individuais; a segunda, pelos proximais, comportamentos e estilos de vida individuais; a terceira é representada pelas redes sociais; a quarta é conhecida como intermediários, a exemplo das condiçóes de vida e trabalho; e, por fim, a camada dos determinantes distais ou macrodeterminantes que compreende as condições econômicas, culturais e ambientais da sociedade. ${ }^{(9)}$

Embora reconheça-se que os DSS podem influenciar na ocorrência da diarreia, acredita-se que mães ou cuidadores com autoeficácia elevada podem minimizar este agravo na infância, pois segundo Bandura, ${ }^{(10)}$ indivíduos com autoeficácia elevada apresentam crenças individuais sobre a capacidade de organizar e executar determinada ação para alcançar um resultado com êxito. Neste sentido, estudo realizado em Fortaleza evidenciou que os filhos cujas mães ou cuidadores tinham autoeficácia elevada, tiveram menos chances de apresentar diarreia. ${ }^{(11)}$

$\mathrm{Na}$ Agenda Nacional de Prioridades de Pesquisa em Saúde, a prevenção primária e secundária da diarreia na infância é um dos temas de destaque. ${ }^{(12)}$ Sabese que em países de baixa renda, crianças menores de três anos são acometidas por, em média, três episódios de diarreia por ano. Este fato tem inúmeras implicaçóes para a saúde da criança, entre as quais a carência de uma nutrição suficiente para o crescimento esperado, o que contribui para uma baixa imunidade e maior adoecimento neste público. ${ }^{(1)}$

Logo, compreender a relação dos determinantes sociais de saúde com a autoeficácia materna na prevenção da diarreia infantil poderá fornecer subsídios e indicadores para realização de açôes que promovam comportamentos maternos saudáveis, além de nortear políticas públicas que minimizem as iniquidades em saúde e, consequentemente, a morbimortalidade por diarreia. Dessa forma, o objetivo do estudo foi 
verificar associação entre os Determinantes Sociais de Saúde com os níveis de autoeficácia materna para prevenção da diarreia infantil.

\section{Métodos}

Estudo descritivo, transversal, realizado a partir da associação dos DSS com a autoeficácia de mães ou cuidadores de crianças menores de cinco anos de idade, conduzido em duas Unidades de Atenção Primária à Saúde (UAPS) do município de Fortaleza, Ceará, Brasil. Fortaleza está dividida em oito Secretarias Regionais (SR), mas, para esse estudo, selecionou-se a SR V, a qual possui índice de desenvolvimento humano (IDH) baixo, variando de 0,135 a 0,395 e a maior ocorrência de diarreia em crianças menores de cinco anos de idade. ${ }^{(5)}$ As duas unidades foram selecionadas por meio de sorteio aleatório simples. Ressalta-se, ainda que para descrição desse estudo seguiu-se as recomendaçôes do checklist Strengthening the Reporting of Observational Studies in Epidemiology - STROBE.

A amostra probabilística foi representativa, sendo determinada com base no cálculo de população finita, ${ }^{(13)}$ adotando-se prevalência igual a $50 \%$, erro amostral de 5\% e coeficiente de confiança de 95\%, contabilizando uma amostra de tamanho "n" igual a 363 mães ou cuidadoras de crianças menores de cinco anos. Apesar disso, algumas variáveis deixaram de serem respondidas por algumas participantes, resultando em números absolutos desiguais no tratamento destes dados.

Para obtenção da amostra, foi levado em consideração os seguintes critérios de inclusão: ser mãe ou cuidador de criança com idade inferior a cinco anos, acompanhada em Unidade de Atenção Primária à Saúde (UAPS). Foram excluídas mães ou cuidadores que apresentavam barreiras de comunicação devido a limitação cognitiva, comprovada por diagnóstico médico, que a impedisse de responder aos instrumentos utilizados para a coleta dos dados; e as(os) que cuidavam de criança com diagnóstico médico de intolerância à lactose, alergia à proteína do leite de vaca ou qualquer outra doença que tenha diarreia como sintoma.
A coleta de dados ocorreu no período de junho a outubro de 2015. As mães ou cuidadores foram individualmente convidados para participarem do estudo no momento em que aguardavam atendimento na UAPS, sendo-lhes apresentados os objetivos do estudo, as orientaçóes detalhadas para a sua participação e as questôes éticas que envolvem a pesquisa com seres humanos. $\mathrm{O}$ consentimento formal foi obtido por meio da assinatura do Termo de Consentimento Livre e Esclarecido.

Em seguida foram direcionados a uma sala reservada, para realizaçáo de entrevista através de dois instrumentos. Cada instrumento levou em média de 15 a 20 minutos para ser respondido. O primeiro instrumento utilizado foi baseado no modelo dos Determinantes Sociais de Saúde, ${ }^{(8)}$ com as seguintes variáveis: camada 1 (determinantes individuais) - faixa etária da máe ou cuidador; camada 2 (determinantes proximais) - estado civil, escolaridade; camada 3 (influência das redes sociais) - número de pessoas no domicílio, ocupação, número de filhos; e camada 4 (determinantes intermediários) - renda familiar, número de filhos, tipo de casa, tipo de piso, destino do lixo, origem da água no domicílio, tipo de sanitário, rede de esgoto, ajuda para cuidar da criança e aleitamento materno exclusivo.

O segundo instrumento foi a Escala de Autoeficácia Para Prevenir A Diarreia Infantil (EAPDI), elaborada no Brasil, com validade e confiabilidade comprovada, ${ }^{(14)}$ apresentando 24 itens, com os seguintes domínios: higiene da família, com 15 itens e práticas alimentares/gerais, com nove itens. A escala é do tipo Likert, com opçôes de resposta que variavam de 1 (discordo totalmente) a 5 (concordo totalmente). A identificação dos níveis de autoeficácia ocorre a partir da soma dos escores, podendo variar de 24 a 120 pontos, considerando-se pontuaçáo igual ou superior a 115 pontos como elevada autoeficácia materna para prevenir a diarreia infantil, e 114 ou menos pontos representando moderada autoeficácia. ${ }^{(11)}$

Os dados obtidos foram organizados em uma planilha do programa Microsoft Excel, por meio de dupla digitação e posterior validação, e exportados ao software Statistical Package for 
Science Social (SPSS), versão 20.0, licença número 10101131007. Para apresentação e análise dos resultados, foi utilizada a estatística descritiva para o cálculo da frequência, em número absoluto e percentual. Os dados foram apresentados em tabelas cruzadas. Fez-se associação entre os Determinantes Sociais de Saúde e a autoeficácia materna para Prevenção da diarreia infantil, por meio do teste de Qui-Quadrado, sendo medida a força das associaçóes pela Razão de Chances (RC) e respectivo intervalo de confiança de $95 \%$. Para essas análises, fixou-se o nível de significância de $5 \%$. Em seguida, fez-se a análise multivariada da regressão logística.

O estudo considerou todos os preceitos éticos da Resolução 466/2012 e da Resolução do COFEN 311/2007, sendo aprovado pelo Comitê de Ética em Pesquisa (CEP), da Universidade Federal do Ceará, Brasil, conforme número 1.116.855.

\section{Resultados}

Os dados apresentados na tabela 1 foram divididos de acordo com as camadas individuais, proximais, influência das redes sociais e intermediárias das mães ou cuidadores das crianças.

Observou-se que, na camada de determinantes individuais, a faixa etária materna se concentrou de 15 a 29 anos (57\%; n=209). Na camada de determinantes proximais, constatou-se que a grande maioria das mães ou cuidadores tinha um companheiro em união estável (79\%; n=284), destas, 167 (59\%) apresentavam autoeficácia moderada, e metade tinha mais de oito anos de estudos (51\%; $\mathrm{n}=186)$. $\mathrm{Na}$ análise da camada de influência das redes sociais, evidenciou-se que a grande maioria era dona de casa e não exercia atividade extradomiciliar (66\%; $\mathrm{n}=237$ ), com quatro ou mais pessoas residindo na mesma casa $(68 \% ; n=246)$, e a maioria possuía mais de três filhos $(88 \% ; n=319)$.

Quanto à camada de determinantes intermediários, metade das mães ou cuidadores tinha renda familiar de um salário mínimo $(50 \% ; \mathrm{n}=172)$, morava em casa de tijolo com reboco $(88 \% ; n=318)$, predominou o consumo da água proveniente da rede pública (99\%; n=357), sanitários com descarga d'água ( $83 \%$; $\mathrm{n}=293)$ e mais da metade não possuía rede de esgoto $(60 \% ; n=218)$. Em relação à saúde da criança, identificou-se que $92 \%(n=332)$ não tinham nenhuma doença e pouco mais da metade ( $51 \% ; n=181)$ das crianças foram amamentadas por menos de seis meses. Além disso, mais da metade (58\%; $\mathrm{n}=208)$ das mães ou cuidadores recebia ajuda de terceiros para cuidar do(a) filho(a).

Constatou-se que $213(58,6 \%)$ das mães ou cuidadores apresentaram autoeficácia moderada, e 150 $(41,3 \%)$ delas, autoeficácia elevada, com a média de 113 escores no somatório na EAPDI.

Verificou-se, na análise da associação entre a autoeficácia materna em prevenir a diarreia infantil com os DSS individuais, significância estatística com a variável faixa etária materna ( $\mathrm{p} 0,017)$, demonstrando que as mães ou cuidadores com idades entre 15 e 29 anos tiveram 1,6 vezes mais chances de apresentar autoeficácia moderada para prevenir a diarreia infantil.

Ao associar a autoeficácia materna em prevenir a diarreia infantil com os DSS proximais (estado civil e escolaridade), não se comprovaram significância estatística entre os DSS influência das redes sociais (o número de pessoas no domicílio, a quantidade de filhos e ocupação) e as chances de apresentarem autoeficácia moderada.

Acerca dos DSS intermediários, as mães ou cuidadores com destino do lixo não realizado pela coleta pública e origem da água do domicílio não proveniente da rede pública tiveram, respectivamente, 3,5 e 2,8 vezes mais chances de apresentarem autoeficácia moderada para prevenir diarreia nas crianças.

Tabela 1. Associação entre os determinantes sociais de saúde e o nível de autoeficácia materna em prevenir a diarreia infantil de mães ou cuidadores de crianças menores de cinco anos

\begin{tabular}{|c|c|c|c|c|}
\hline \multicolumn{5}{|c|}{ Autoeficácia materna } \\
\hline DSS & $\begin{array}{c}\text { Moderada } \\
\mathrm{n}(\%)\end{array}$ & $\begin{array}{c}\text { Elevada } \\
\mathrm{n}(\%)\end{array}$ & $\begin{array}{c}\mathrm{RC} \\
(\mathrm{IC} 95 \%)\end{array}$ & $p$-value $e^{\star \star}$ \\
\hline \multicolumn{5}{|l|}{ Individuais } \\
\hline $\begin{array}{l}\text { Faixa etária da mãe ou cuidador } \\
\text { (anos) }(n=363)\end{array}$ & & & & 0,017 \\
\hline $15-29$ & $133(63,6)$ & $76(36,4)$ & $1,6(1,0-2,4)$ & \\
\hline $30-55$ & $80(51,9)$ & $74(48,1)$ & & \\
\hline \multicolumn{5}{|l|}{ Determinantes proximais } \\
\hline Estado civil $(n=360)$ & & & & 0,529 \\
\hline Solteira & $45(59,2)$ & $31(40,8)$ & $1,0(0,6-1,7)$ & \\
\hline Casada & $167(58,8)$ & $117(41,2)$ & & \\
\hline
\end{tabular}

Continua. 
Continuação.

\begin{tabular}{|c|c|c|c|c|}
\hline \multicolumn{5}{|c|}{ Autoeficácia materna } \\
\hline DSS & $\begin{array}{l}\text { Moderada } \\
\mathrm{n}(\%)\end{array}$ & $\begin{array}{c}\text { Elevada } \\
\mathrm{n}(\%)\end{array}$ & $\begin{array}{c}\text { RC } \\
\text { (IC95\%) }\end{array}$ & $p$-value ${ }^{\star \star}$ \\
\hline Escolaridade ( $n=362)$ & & & & 0,369 \\
\hline$\leq 8$ & $101(57,4)$ & $75(42,6)$ & $0,9(0,5-1,3)$ & \\
\hline$>8$ & $111(59,7)$ & $75(40,3)$ & & \\
\hline Influência das redes sociais & & & & \\
\hline $\begin{array}{l}\text { Números de pessoas no domicílio } \\
(\mathrm{n}=359)\end{array}$ & & & & 0,527 \\
\hline De 4 ou mais & $143(58,1)$ & $103(41,9)$ & $0,9(0,6-1,5)$ & \\
\hline$\leq 3$ & $66(58,4)$ & $47(41,6)$ & & \\
\hline Ocupação (n=357) & & & & 0,184 \\
\hline Outro & $66(55,0)$ & $54(45,0)$ & $0,7(0,5-1,2)$ & \\
\hline Do lar & $144(60,6)$ & $93(39,4)$ & & \\
\hline $\mathrm{N}^{\circ}$ de filhos ( $\left.\mathrm{n}=361\right)$ & & & & 0,540 \\
\hline$>3$ & $188(58,9)$ & $131(41,1)$ & $0,9(0,5-1,8)$ & \\
\hline$\leq 3$ & $25(59,5)$ & $17(40,5)$ & & \\
\hline Intermediários & & & & \\
\hline $\begin{array}{l}\text { Renda familiar (salário mínimo) } \\
(\mathrm{n}=343)\end{array}$ & & & & 0,270 \\
\hline Até 1,0 & $104(60,8)$ & $67(39,2)$ & $1,1(0,7-1,8)$ & \\
\hline 2 ou mais & $98(57,0)$ & $74(43,0)$ & & \\
\hline Sexo da criança ( $n=363$ ) & & & & 0,246 \\
\hline Masculino & $106(56,7)$ & $81(43,3)$ & $0,8(0,5-1,2)$ & \\
\hline Feminino & $107(60,8)$ & $69(39,2)$ & & \\
\hline Criança estuda ( $\mathrm{n}=357$ ) & & & & 0,491 \\
\hline Sim & $67(57,8)$ & $49(42,2)$ & $0,9(0,6-1,5)$ & \\
\hline Não & $141(58,5)$ & $100(41,5)$ & & \\
\hline Tipo de casa (reboco) (n=362) & & & & 0,337 \\
\hline Sem & $24(54,5)$ & $20(45,5)$ & $0,8(0,4-1,5)$ & \\
\hline Com & $188(59,1)$ & $130(40,9)$ & & \\
\hline Tipo de piso (n=362) & & & & 0,190 \\
\hline Cimento & $94(61,8)$ & $58(38,2)$ & $1,2(0,8-1,8)$ & \\
\hline Cerâmica & $119(56,7)$ & $91(43,3)$ & & \\
\hline Destino do lixo ( $n=362)$ & & & & 0,193 \\
\hline Outros & $5(83,3)$ & $1(16,7)$ & $3,5(0,4-30,7)$ & \\
\hline Coleta & $208(58,4)$ & $148(41,6)$ & & \\
\hline $\begin{array}{l}\text { Origem da água do domicílio } \\
(\mathrm{n}=362)\end{array}$ & & & & 0,316 \\
\hline Outros & $4(80,0)$ & $1(20,0)$ & $2,8(0,3-25,5)$ & \\
\hline Rede pública & $209(58,5)$ & $148(41,5)$ & & \\
\hline $\begin{array}{l}\text { Tipo de sanitário (descarga) } \\
(\mathrm{n}=354)\end{array}$ & & & & 0,302 \\
\hline Sem & $38(62,3)$ & $23(37,7)$ & $1,2(0,6-2,1)$ & \\
\hline Com & $169(57,7)$ & $124(42,3)$ & & \\
\hline Possui rede de esgoto ( $n=361$ ) & & & & 0,373 \\
\hline Não & $130(59,6)$ & $88(40,4)$ & $1,0(0,7-1,6)$ & \\
\hline Sim & $82(57,3)$ & $61(42,7)$ & & \\
\hline $\begin{array}{l}\text { Recebe ajuda para cuidar da } \\
\text { criança }(n=361)\end{array}$ & & & & 0,445 \\
\hline Sim & $121(58,2)$ & $87(41,8)$ & $0,9(0,6-1,4)$ & \\
\hline Não & $91(59,5)$ & $62(40,5)$ & & \\
\hline $\begin{array}{l}\text { Criança tem alguma enfermidade } \\
(n=360)\end{array}$ & & & & 0,447 \\
\hline Sim & $17(60,7)$ & $11(39,3)$ & $1,1(0,5-2,4)$ & \\
\hline Não & $193(58,1)$ & $139(41,9)$ & & \\
\hline $\begin{array}{l}\text { Aleitamento materno exclusivo } \\
(\mathrm{n}=356)\end{array}$ & & & & 0,108 \\
\hline$<6$ meses & $112(61,9)$ & $69(38,1)$ & $1,3(0,8-2,0)$ & \\
\hline 6 meses ou mais & $96(54,9)$ & $79(45,1)$ & & \\
\hline
\end{tabular}

*Valor do salário mínimo na época da coleta dos dados: $\mathrm{R} \$ 880,00$; ** $p$-value. Testes Exato de Fisher $\mathrm{e}$ Razão de verossimilhança

\section{Discussão}

Os DSS influenciam o processo saúde-doença, de forma diferente, cada um dos grupos sociais, havendo, portanto, indivíduos em condiçóes mais vulneráveis. ${ }^{(15)}$ Logo, é necessário compreender o contexto socioeconômico e as condiçóes ambientais em que as pessoas vivem, além dos costumes sociais e culturais para o planejamento de intervençóes. ${ }^{(16)}$

Além disso, deve-se considerar também a autoeficácia, uma vez que é um potencial preditor para comportamentos promotores de saúde, podendo potencializar ou limitar cuidados maternos à criança, ${ }^{(16)}$ além de contribuir na prevenção e no controle da diarreia infantil, pois mães com conhecimento adequado e autoeficácia elevada tem maior probabilidade de praticar o cuidado diário ao filho. ${ }^{(14)}$ Assim, é oportuno identificar os DSS que podem influenciar na autoeficácia materna, e, por conseguinte, implementar estratégias que atuem buscando elevar este aspecto. ${ }^{(17)}$

Conforme o modelo Dahlgreen e Whithead (2007), os determinantes individuais e não modificáveis, como idade, sexo e fatores genéticos, exercem influência sobre condiçóes de saúde. ${ }^{(8)}$ Neste estudo, observou-se maior prevalência de mães ou cuidadores entre 15 e 29 anos de idade, as quais tiveram mais chances de apresentarem autoeficácia moderada, em detrimento da autoeficácia elevada. Corroborando com estes dados, estudo desenvolvido em Fortaleza-CE, com mães de crianças menores de cinco anos, evidenciou que esta variável pode influenciar nos níveis de autoeficácia que as mães apresentam, sobretudo, na prevenção da diarreia. Em consonância, pesquisa realizada na Indonésia, também, demonstrou que a idade materna foi estatisticamente associada com a ocorrência da diarreia em crianças. ${ }^{(18)}$ Deste modo, acredita-se que a idade mais avançada torna o cuidador mais experiente, podendo melhorar as práticas de higiene e alimentação da criança, e, consequentemente, sentir-se mais confiante para prevenir a diarreia.

Com relação aos DSS proximais que correspondem aos modificáveis, como o comportamento e os estilos de vida individuais, observou-se que a maioria das mães ou cuidadores tinha um companhei- 
ro(a) mantendo união estável, e metade tinha mais de oito anos de estudos. Semelhante a esses achados, estudo realizado na Indonésia identificou associação estatisticamente significativa entre escolaridade materna e a ocorrência de diarreia, de modo que a mãe possuir baixa escolaridade pode limitar o conhecimento acerca da prevençáo das doenças diarreicas, constituindo-se em fator de risco para o adoecimento dos filhos. ${ }^{(19)}$

Estudo transversal, realizado em Bankura, na Índia, com 76 mães de crianças menores de cinco, constatou que quanto mais elevada a escolaridade materna, melhores são os conhecimentos relacionados ao manejo da diarreia infantil. ${ }^{(20)}$ Embora não se tenha encontrado significância na associação entre estado civil e escolaridade com a autoeficácia materna, estudo longitudinal, realizado em Fortaleza, com 90 mães de crianças menores de cinco anos de idade, comprovou que o estado civil e a escolaridade apresentaram significância com os níveis de autoeficácia materna para prevenir a diarreia infantil. ${ }^{(21)}$

Em relação aos DSS de influência das redes sociais (ocupação, número de pessoas no domicílio e número de filhos), que demonstra que o nível de coesão social é relevante para saúde da populaçáo, ${ }^{(8)}$ evidenciou-se prevalência de mães ou cuidadores donas de casa, com quatro ou mais pessoas residindo no mesmo domicílio, e a maioria possuía mais de três filhos. Embora no presente estudo não se tenha identificado associação considerável sobre a ocupação e a autoeficácia materna, pesquisa realizada no Peru identificou que o fato de a mãe exercer trabalho extradomiciliar poderia prejudicar os cuidados à criança, pois estas apresentavam menor tempo disponível com as crianças. ${ }^{(22)}$ Contrapondo, investigação realizada em Fortaleza identificou que as mães que trabalhavam, sentiam-se mais capazes de tomar os cuidados de manejo e prevenção da diarreia na infância. ${ }^{(11)}$ Diante disto, acredita-se que a ocupação da mãe pode influenciar na autoconfiança destas em prevenir a diarreia.

Sobre o número de pessoas na mesma residência, estudo desenvolvido no Paquistáo apontou que ambientes com aglomeraçóes de pessoas apresentam maior probabilidade da presença de geohelmintíases, por conseguinte, as crianças que compar- tilham o mesmo quarto com mais de três pessoas apresentaram maior risco de desenvolver diarreia. (23) Ademais, pesquisa no Senegal certificou que a presença de dois ou mais filhos com menos de cinco anos de idade que vivem no mesmo domicílio esteve relacionado à ocorrência de diarreia. ${ }^{(24)}$

Acerca do DSS referente ao número de filhos, destaca-se que quanto menor o número de filhos, mais atenção e dedicação será dada à criança, e, portanto, a mãe e/ou cuidador se sentirá capaz de realizar adequadamente os cuidados necessários. ${ }^{(11)}$ Acrescentase, ainda, pesquisa realizada em dois municípios nordestinos que sinalizou que as mães com maior número de filhos procuravam menos os serviços de saúde. ${ }^{(25)}$ Este dado pode ser explicado pelo fato de estas mães possuírem mais experiência para realizar cuidados à criança, necessitando de menor busca de atendimento hospitalar. Ainda, pesquisa realizada no interior do Ceará evidenciou que as mães com três ou mais filhos tinham crianças com mais episódios diarreicos. ${ }^{(26)}$ Percebe-se, assim, que o número de filhos pode ser fator protetor ou de risco. Contudo, independente da quantidade de filhos, é importante que o cuidado ofertado à criança seja adequado para prevenir a diarreia e que caso esta ocorra a família deve procurar um serviço de saúde.

Aponta-se que os DSS intermediários, analisados no presente estudo, estavam relacionados, principalmente, ao contexto sanitário das famílias estudadas, e mesmo não tendo encontrado associação estatisticamente significante com os níveis de autoeficácia materna, constatou-se que as mães tiveram mais chances de apresentar autoeficácia moderada em detrimento da elevada, quando a origem da água era proveniente de outra fonte e não da rede pública de abastecimento e quando náo tinham o destino do lixo realizado pela coleta pública.

Sabe-se que a carência de saneamento adequado é tida como uma das principais causas da contaminação das águas para o abastecimento humano e, portanto, contribui para os casos de doenças de veiculação hídrica. ${ }^{(27)}$ Assim, melhorias na qualidade da água, no saneamento básico e nas condiçóes de higiene da populaçáo poderiam reduzir os casos de doenças diarreicas, sobretudo, em grupos etários mais vulneráveis, como crianças. ${ }^{(28)}$ 
Os DSS intermediários que corresponde ao tipo de casa e tipo de piso estáo relacionados diretamente às condiçóes de moradia das famílias, e tem impacto na saúde humana, pois estima-se que os mais vulneráveis, como crianças, passem 80 a $90 \%$ do tempo no domicílio. ${ }^{(29)}$

Estudo realizado no Ceará evidenciou que morar em casa de tijolo com reboco e piso de cerâmica ou cimento teve correlação significativa com a autoeficácia materna para prevenção da diarreia infantil. ${ }^{(16)}$ De modo similar, estudo transversal realizado no Município de Guarulhos, em São Paulo, Brasil, afirma que o fato de a casa ser feita de tijolos com reboco facilita limpeza adequada das mesmas, promovendo a prevenção da diarreia, consequentemente, contribuindo para autoeficácia materna na prevenção da doença. ${ }^{(30)}$

Em relação ao DSS intermediário renda familiar, também não apresentou correlação estatística com a autoeficácia entre as mães e/ou cuidadores. No entanto, estudo que buscou associar as condiçôes socioeconômicas com a autoeficácia materna para prevenir a diarreia infantil, identificou que quanto menor a renda familiar, menores os níveis de autoeficácia materna. ${ }^{(21)}$ Desta forma, percebe-se que a renda é um DSS que repercute sobre o estado de saúde dos indivíduos, pois permite atender às necessidades básicas de uma família. Neste aspecto, pesquisa realizada em Fortaleza evidenciou que as mães que possuem renda familiar mais elevadas têm melhores condições de vida e habitação, podendo estes fatores influenciarem na autoconfiança das mesmas em prevenir diarreia infantil. ${ }^{(11)}$

Acerca da amamentação exclusiva, no que diz respeito aos DSS intermediários, pesquisas apontam o aleitamento materno como fator protetor para diarreia em crianças, conforme estudo prospectivo de coorte realizado no Vietná, em 2012, com 1.049 lactentes para investigar fatores pré-natais e precoces de doenças, como diarreia e pneumonia, percebeu que a chance de uma criança se internar por diarreia diminuiu em mais $60 \%$, devido à prática do aleitamento materno exclusivo. ${ }^{(31)}$

Os fatores que influenciam a amamentação são multidimensionais, envolvendo questóes sociais, econômicas, culturais e psicológicas. Portanto, acre- dita-se que filhos(as) de mães com autoeficácia elevada têm mais chances de receberem o leite materno exclusivo por seis meses.

Enfatiza-se que a camada de determinantes distais ou macrodeterminantes não foi integrada a esta pesquisa, contudo, esses determinantes são relevantes por envolverem dimensóes social, cultural e econômica, onde se manifesta no ambiente em que indivíduo está inserido. Além disso, esses aspectos possibilitam identificar sua condição de saúde, reivindicar seus direitos legais, planejar açóes a serem desenvolvidas, objetivando a integralidade e a universalidade da assistência, como princípio de justiça social, além de promover um desenvolvimento sustentável, reduzindo as desigualdades sociais e econômicas, as violências, a degradação ambiental e seus efeitos sobre a sociedade. ${ }^{(8)}$

Ressalta-se que o Índice de Desenvolvimento Humano (IDH) dos bairros da Secretaria Regional $\mathrm{V}$, como dito anteriormente, é baixo. Segundo o Instituto de Pesquisa e Estratégia Econômica do Ceará (IPECE), dos dez bairros de Fortaleza-CE com menor renda, seis estão localizados na SER $\mathrm{V} .{ }^{(32)}$ Portanto, é fundamental que o gestor municipal também tenha o conhecimento da influência dos fatores sociais, comportamentais, culturais e econômicos na vida das pessoas, para um melhor planejamento de suas açóes, tornando mais abrangente a sua atuação na atenção à saúde da população.

Este estudo sinaliza que para promover a saúde da criança e prevenir a diarreia, aspectos como os determinantes sociais da saúde e a autoeficácia das mães ou cuidadores precisam ser considerados. Ademais, para entender o contexto no qual a criança está inserida, estas devem ser estudadas a partir de ângulo multidimensional, para que as intervençôes em saúde possam ser mais eficazes, com intuito de diminuir as hospitalizaçóes e a morbimortalidade por diarreia em crianças menores de cinco anos.

$\mathrm{O}$ estudo teve como limitação a utilização do desenho transversal, que não permitiu associação dos DSS e a autoeficácia materna para prevenir a diarreia infantil ao longo do tempo. Assim, recomenda-se que outros estudos sejam realizados com maior tempo de acompanhamento, com análise de todas as camadas dos DSS, além de outras variáveis. 
Espera-se que os dados apresentados nesse estudo contribuam para atuação dos profissionais de saúde na atenção primária, pois entender os DSS e o modo como interferem nas condições de saúde é um importante meio de avaliar a capacidade de manutenção das condiçôes de saúde de um indivíduo, além de fortalecer e direcionar um plano de cuidados de forma estratégica, considerando os determinantes sociais, as demandas da população e necessidades dos seus territórios. Assim, faz-se premente oferecer subsídios para realizaçáo de açôes educativas que promovam comportamentos maternos saudáveis a fim de minimizar a morbimortalidade por diarreia, e orientar seus pacientes a buscarem seus diretos por meios legais, e propor a implementação de medidas e mudanças no próprio sistema de saúde onde está inserido de acordo com suas peculiaridades.

\section{Conclusão}

A utilização da EAPDI permitiu identificar os níveis de autoeficácia materna para prevenir a diarreia infantil e verificar a associação com o DSS. Foram observadas associaçóes estatísticas entre a autoeficácia materna e o DSS individual: faixa etária materna. Verificou-se que as mães com idade de 15 a 29 anos tiveram mais chances de apresentarem autoeficácia moderada para prevenir a diarreia infantil. Apesar dos DSS intermediários não apresentaram associação significante neste estudo, as mães ou cuidadores com os DSS destino do lixo e origem da água do domicílio inadequados foram as que mais tiveram chances de apresentarem autoeficácia moderada. Estudos dessa natureza são pertinentes para entender o contexto no qual a criança está inserida, possibilitando, assim, intervençôes mais efetivas. Ademais, diante do conhecimento sobre a associação dos DSS e autoeficácia materna, cabe aos enfermeiros uma maior ênfase em orientar as mães ou cuidadores de crianças sobre a prevenção da diarreia e promover açôes educativas que visem aumentar a autoeficácia das mesmas em prevenir a doença, mesmo quando inseridas em realidades menos favorecidas.

\section{Agradecimentos}

Às agências de financiamento do Programa de Pesquisa para o SUS: Gestão Compartilhada em Saúde - PPSUS (Número de identificação do processo: PP3-0118-00121.01.00/17 e SPU: 3926819/2017) Decit/SCTIE/MS por intermédio do Conselho Nacional de Desenvolvimento Científico e Tecnológico (CNPq) e apoio da Fundação Cearense de Apoio ao Desenvolvimento Científico e Tecnológico (FUNCAP) e Secretaria de Saúde do Estado do Ceará - SESA; e às mães que participaram do estudo.

\section{Colaborações}

Mendes ERR, Paula PHA, Lima KF, Almeida PC, Pinheiro PNC, Melo ESJ e Barbosa LP contribuíram com a concepção do estudo, análise e interpretação dos dados, redação do artigo, revisão crítica relevante do conteúdo intelectual e aprovação da versão final a ser publicada.

\section{Referências}

1. World Health Organization (WHO). Diarrhoeal Disease. Geneva: WHO; 2019 [cited 2020 Sep 05]. https://www.who. int/news-room/factsheets/detail/diarrhoeal-disease

2. Brasil. Ministério da Saúde. Secretaria de vigilância em saúde. Doenças diarreicas agudas [Internet]. Brasília (DF): 2018 [citado 2018 Mai 23]. Disponível em: http://portalarquivos2.saude.gov.br/images/pdf/2018/ maio/09/Apresenta----0-DDA-Abril-2018.pdf

3. Brasil. Ministério da Saúde. Departamento de informática do Sistema Único de Saúde (DATASUS). Sistema de Informações Hospitalares do SUS. Sistema De Informação De Atenção Básica. Situação de saúde [Internet]. Brasília (DF): Ministério da Saúde; 2018 [citado 2018 Abr 23]. Disponível em: http://tabnet.datasus.Gov.br/cgi/deftohtm. exe?siab/cnv/siabsce.def

4. Ceará. Governo do Estado do Ceará. Secretaria da Saúde. Boletim epidemiológico de mortalidade: materna, infantil e fetal. Fortaleza (CE): Secretaria da Saúde; 2017 [citado 2020 Set 14]. Disponível em: https://www.saude.ce.gov.br/wp-content/uploads/sites/9/2018/06/ boletim_mortalidade_06_04_2017.pdf

5. Fortaleza. Secretaria Municipal de Saúde. Sistema de Monitoramento Diário de Agravos (SIMDA). Diarreia: casos por bairro de residência segundo a faixa etária, fortaleza, 2017. Fortaleza: Secretaria Municipal de Saúde; 2019 [citado 2019 Set 13]. Disponível em: http://tc1.sms.fortaleza.ce.gov.br/simda/notificacao/faixa?agravo= a09\&ano=2017\&faixaetaria $=2 \&$ modo=bairro\&regional $=$ 
6. Rouquayrol MZ, Goldbaum M, Santana EW. Epidemiologia, história natural e prevenção de doenças. In: Rouquayrol MZ, Gurgel M. Epidemiologia e Saúde. 7a ed. Rio de Janeiro: MedBook; 2013.

7. Solomon LS, Kanter MH. Health care steps up to social determinants of health: current context. Perm J. 2018;22:8-139.

8. Buss PM, Pellegrini FA. A saúde e seus determinantes sociais. Physis: Rev Saúde Coletiva. 2007;17(1):77-93.

9. Dahlgren $\mathrm{G}$, Whitehead M. Policies and strategies to promote social equity in health. Background document to WHO - strategy paper for Europe. Stockolm: Arbetsrapport/Institutet för Framtidsstudier; 2007 [cited 2017 Apr 23]. Available from: https://core.ac.uk/download/ pdf/6472456.pdf

10. Bandura A. On the functional properties of perceived self-efficacy revisited. J Management. 2012;38(1):9-44.

11. Joventino ES, Bezerra KC, Coutinho RG, Almeida PC, Oriá MO, Ximenes LB. Condições sociodemográficas e sanitárias na autoeficácia materna para prevenção da diarreia infantil. Rev Salud Pública. 2013;15(4):54254.

12. Brasil. Ministério da Saúde. Departamento de informática do Sistema Único de Saúde (DATASUS). Óbitos por causas evitáveis em menores de 5 anos - Ceará. Ceará: Ministério da Saúde; 2015 [citado 2018 Abr 23]. Disponível em: http://tabnet.datasus.gov.br/cgi/tabcgi.exe?sim/ cnv/evita10CE.def

13. Hulley SB, Cummings SR, Brower WS. Delineando a pesquisa clínica. 4a ed. Porto Alegre: Artmed; 2015.

14. Joventino ES, Ximenes LB, Penha JC, Andrade LC, Almeida PC. The use of educational video to promote maternal self-efficacy in preventing early childhood diarrhoea. Int J Nurs Pract. 2017;23(3).

15. Jorge BM, Fumincelli L, Souza-Junior VD, Almeida RG, Mazzo A Ventura CA, et al. Social Determinants of Health in the lives of urinary catheter users. Rev Bras Enferm. 2018;71(4):1928-33.

16. Oliveira RK, Oliveira BS, Bezerra JC, Silva MJ, Melo FM, Joventino MS Influence of socio-economic conditions and maternal knowledge in self-effectiveness for prevention of childhood diarrhea. Esc Anna Nery. 2017;21(4):e2016036.

17. Sabino LM, Ferreira AM, Mendes ER, Joventino ES, Gubert FA, Penha $\mathrm{JC}$, et al. Validation of primer for promoting maternal self-efficacy in preventing childhood diarrhea. Rev Bras Enferm. 2018;71(Suppl 3):1412-9

18. Suyitno S, Chompikul J, Tiraphat S, Anye KS. Prevalence and risk factors for diarrhea among children aged less than two years in Central Kalimantan Province, Indonesia. J Pub Health Development. 2019;17(1):31-45.

19. Cronin AA, Sebayang SK, Torlesse H, Nandy R. Association of safe disposal of child feces and reported diarrhea in indonesia: need for stronger focus on a neglected risk. Int J Environ Res Public Health. 2016;13(3):310.
20. Gazi E, Chowdhury A, Kumar R, Sarkar AP, Basu SS, Saha S. Can mothers care for acute diarrhoeal disease of their under five children effectively at home? a cross sectional study in slum community in bankura. J Evidence Based Med Healthcare. 2015;2(36):5575-5584.

21. Andrade LC, Mendes ER, Vasconcelos IA, Joventino ES, Almeida PC, Ximenes LB. Socio-demographic factors relating to mothers' selfefficacy in preventing childhood diarrhea: a longitudinal study. Online Braz J Nurs. 2015;14(1):62-70.

22. Pantenburg B, Ochoa TJ, Ecker L, Ruiz J. Feeding of Young children during diarrhea: caregivers' intended practices and perceptions. Am J Trop Med Hyg, 2014;91(3):555-62.

23. Siziya S, MuulaAS, Rudatsikira E. Correlates of diarrhoea among children below the age of 5 years in Sudan. Afr Health Sci. 2013;13(2):376-83.

24. Thiam S, Diène AN, Fuhrimann S, Winkler MS, Sy I, Ndione JA, et al. Prevalence of diarrhoea and risk factors among children under five years old in Mbour, Senegal: a cross-sectional study. Infect Dis Poverty. 2017;6(1):109.

25. Costa JS, Cesar JA, Weber AP, Garcez AS, Nora CR, Rower HB, et al. Características das crianças menores de cinco anos atendidas em serviços de atenção básica em dois municípios do nordeste brasileiro. Rev Bras Saúde Matern Infant. 2015;15(1):33-46.

26. Oliveira B, Oliveira SB, Bezerra RK, Melo JC, Sousa FM, Monteiro FP, et al. Social conditionsand maternal conducts in the prevention and management of infantile diarrhea. Cogitare Enferm. 2017;224:e50294.

27. Ercumen A, Naser AM, Unicomb L, Arnold BF, Colford JM Jr, Luby SP. Effects of source- versus household contamination of tubewell water on child diarrhea in rural Bangladesh: a randomized controlled trial. PLoS One. 2015;10(3):e0121907.

28. Bhutta ZA, Das JK, Walker N, Rizvi A, Campbell H, Rudan I, Black RE; Lancet Diarrhoea and Pneumonia Interventions Study Group. Interventions to address deaths from childhood pneumonia and diarrhoea equitably: what works and at what cost? Lancet. 2013;381(9875):1417-29.

29. Pasternak S. Habitação e saúde. Estudos Avançados. 2016;30(86):51-66.

30 Paz MG, Almeida MF, Gunther WM. Diarrhea in children and sanitation and housing conditions in periurban areas in the city of Guarulhos, SP. Rev Bras Epidemiol. 2012;15(1):188-97.

31. Hanieh S, Ha TT, Simpson JA, Thuy TT, Khuong NC, Thoang DD, et al. Exclusive breast feeding in early infancy reduces the risk of inpatient admission for diarrhea and suspected pneumonia in rural Vietnam: a prospective cohort study. BMC Public Health. 2015;15:1166.

32. Ceará. Governo do Estado do Ceará. Secretaria do Planejamento e Gestão (SEPLAG). Instituto de Pesquisa e Estratégia Econômica do Ceará (IPECE). Perfil Municipal de Fortaleza. Tema VII: Distribuição Espacial da Renda Pessoal. Fortaleza (CE): SEPLAG; IPECE; 2012 [citado 2021 Fev 13]. Disponível em: https://www.ipece.ce.gov.br/wp-content/uploads/ sites/45/2012/12/Ipece_Informe_47_03_dezembro_2012.pdf 